\title{
9 QUELLENVERZEICHNIS
}

\subsection{Literaturverzeichnis}

ACCORD (Austrian Centre for Country of Origin \& Asylum Research and DocumenTATION) (2016), Das Schulsystem in Syrien. <https://www.roteskreuz.at/uploads/media/ Schulsystem_Syrien_2016.pdf $>$ [Zugriff: 25.05.2020].

Ager A. \& Strang A. (2008), Understanding Integration. A Conceptual Framework. Journal of Refugee Studies 21 (2), pp. 166-191.

Ager A. \& Strang A. (2010), Refugee Integration: Emerging Trends and Remaining Agendas. Journal of Refugee Studies 23 (4), pp. 589-607.

Aigner A. (2018), Housing Entry Pathways of Refugees in Vienna, a City of Social Housing. Housing Studies 34 (5), pp. 779-803.

Altrichter H. \& Feyerer E. (2017), Schulentwicklung und Inklusion in Österreich. In: LüTJeKlose B., Miller S. Schwab S. \& Streese B. (eds.), Inklusion: Profile für die Schul- und Unterrichtsentwicklung in Deutschland, Österreich und der Schweiz. Theoretische Grundlagen - Empirische Befunde - Praxisbeispiele. Münster, Waxmann Verlag, pp. 31-42.

Amt DER NÖ LANDESREgIerung (2020), Gemeindedaten. <http://www.noe.gv.at/noe/ZahlenFakten/Statistik-Gemeinden.html> [Zugriff: 05.08.2020].

AMT DER OÖ LANDESREGIERUNG (2019), Bildung als Integrationsmotor in der Stadt. Oberösterreich praktiziert Integration ab dem ersten Tag der Anwesenheit. Information zur Pressekonferenz mit Integrations-Landesrat Rudi Anschober und Bildungsstadträtin Mag. ${ }^{a}$ Eva Schobesberger, 4. April 2019, Linz. <https://www.land-oberoesterreich.gv.at/Mediendateien/LK/PKAnschober442019Internet.pdf> [Zugriff: 24.09.2020].

Amtsblatt St. ANDRÄ-WöRdern (2016), Kommentar von Bürgermeister Maximilian Titz. 05/2016, p. 3. <https://www.staw.at//system/web/GetDocument.ashx?fileId=1231715> [Zugriff: 04.03.2019].

AmtsBlatt St. Andrä-WöRdern (2017), Neustart in der Dammstraße. 04/2017, p. 27. <https:// www.staw.at//system/web/GetDocument.ashx?fileId=1340407> [Zugriff: 06.03.2019].

Anthias F. (2013), Moving Beyond the Janus Face of Integration and Diversity Discourses: Towards an Intersectional Framing. The Sociological Review 61 (1), pp. 323-343.

APA-OTS (2018), Landesrat Waldhäusl zur Integrationsreferenten-Konferenz: Ja zu Niederösterreich-Anträgen (21.06.2018). <https://www.ots.at/presseaussendung/OTS_20180621 OTS0175/landesrat-waldhaeusl-zur-integrationsreferenten-konferenz-ja-zu-niederoesterreich-antraegen> [Zugriff: 20.07.2019].

AsYlKoORDINATION ÖsterReICH (2018), Grundversorgung (asylKoordinaten - Infoblatt der Asylkoordination Österreich 2). <www.asyl.at/adincludes/dld.php?datei=12.02. ma,asylkoordinaten_1_2019_web_neu2.pdf.> [Zugriff: 26.03.2020].

Ateş G. \& Reinprecht C. (2017), Reziproke Lerneffekte: Einsichten aus einem europäischen Projekt der Erwachsenenbildung mit ethnischen Minderheiten und MigrantInnen. In: Altenburg F., Faustmann A., Pfeffer T. \& Skrivanek I. (eds.), Migration und Globalisierung in Zeiten des Umbruchs - Festschrift für Gudrun Biffl. Edition Donau-Universität Krems, pp. 301-314. 
BAUBÖCK R. (2001), Integration von Einwanderern - Reflexionen zum Begriff und seinen Anwendungsmöglichkeiten. In: WaldRAuCh H. (ed.), Die Integration von Einwanderern: Ein Index der rechtlichen Diskriminierung. Frankfurt am Main: Campus Verlag, pp. 27-52.

BeCKer B. (2010), Bildungsaspirationen von Migranten. Determinanten und Umsetzung in Bildungsergebnisse. Mannheimer Zentrum für Europäische Sozialforschung, Working Paper 137, Universität Mannheim.

Behrensen B. \& Westphal M. (2009), Junge Flüchtlinge - ein blinder Fleck in der Migrationsund Bildungsforschung. Bildung junger Flüchtlinge als Randthema in der migrationspolitischen Diskussion. In: Krappmann L., Lob-HüDEPohl A., Bohnmeyer A. \& KurzKeMAasmeier S. (eds.), Bildung für junge Flüchtlinge - ein Menschenrecht: Erfahrungen, Grundlagen und Perspektiven. Bielefeld, W. Bertelsmann Verlag, pp. 45-58.

BENDL A. (2014), AsylwerberInnen in Niederösterreich - Integration (nicht) erwünscht? Masterarbeit Universität Wien.

Benholz C., Frank M. \& Niederhaus C. (2016), Neu zugewanderte Schülerinnen und Schüler - eine Gruppe mit besonderen Potentialen. Beiträge aus Forschung und Schulpraxis. Münster, Waxman Verlag.

BezirksblätTER (2017), 21 Einsätze in Asylheim. 09.06.2017. <https://www.meinbezirk.at/tulln/ politik/21-einsaetze-in-asylheim-d2152042.html.> [Zugriff: 07.07.2019].

BIERwIRTH A. (2017), Erwachsenenbildung in der Einwanderungsgesellschaft. Anforderungen an Beschäftigte einer Berliner Volkshochschule vor dem Hintergrund der Zuwanderung Asylsuchender. Erwachsenenpädagogischer Report, Band 58. Humboldt-Universität zu Berlin. <https://edoc.hu-berlin.de/handle/18452/19892> [Zugriff: 05.06.2020].

BIFFL G. (2017), Daten und Fakten zur Flüchtlingszuwanderung: Herausforderungen für das Erwachsenen-Bildungssystem und Erwerbssystem. Magazin erwachsenenbildung.at 31 (1), pp. 16-29. <https://erwachsenenbildung.at/magazin/17-31/meb17-31.pdf> [Zugriff: 07.04.2020].

Biffl G., Kilic H. \& ZentNer M. (2019), Einstellungen, Erwartungen und Ressourcen weiblicher Flüchtlinge. Grundlagenstudie zu den tatsächlich wahrnehmbaren Möglichkeiten von Frauen mit Fluchterfahrung. Department für Migration und Globalisierung, Donau-Universität Krems.

BIFIE(2020), Häufiggestellte Fragen zur Überprüfung der Bildungsstandards. $<$ https://www.bifie. at/haeufig-gestellte-fragen-zur-ueberpruefung-der-bildungsstandards/> [Zugriff: 09.06 . 2020].

BINDER S. (2017), Sozialanthropologische Flüchtlingsforschung: Begriffsbestimmungen, Konzepte und theoretische Perspektiven. In: Binder S. \& FrataceK G. (eds.), Facetten von Flucht aus dem Nahen und Mittleren Osten. Wien, Facultas, pp. 124-149.

BMFSFJ (Bundesministerium für Familie, Senioren, Frauen und Jugend) (ed.) (2017), Zweiter Bericht über die Entwicklung des bürgerschaftlichen Engagements in der Bundesrepublik Deutschland. Schwerpunktthema: „Demographischer Wandel und bürgerschaftliches Engagement: Der Beitrag des Engagements zur lokalen Entwicklung “. Berlin.

Böhm W. \& Streihammer J. (2016), Die Aufarbeitung der Kölner Silvesternacht. Die Presse, 06.01.2016. <https://diepresse.com/home/ausland/welt/4899178/Die-Aufarbeitung-derKoelner-Silvesternacht> [Zugriff: 24.08.2019].

Breit S., Eder F., Krainer K., Schreiner C., Seel A. \& Spiel C. (eds.) (2019), Nationaler Bildungsbericht Österreich, Band 2, Fokussierte Analysen und Zukunftsperspektiven für das Bildungswesen. Bundesministerium für Bildung, Wissenschaft und Forschung 
/ Bundesinstitut für Bildungsforschung, Innovation \& Entwicklung des österreichischen Schulwesens.

Brückner W., Evers J., Nowak C., SchlöGl P. \& Veichtlbauer J. (2017), Erwachsenengerechter Pflichtschulabschluss (ePSA). <https://erwachsenenbildung.at/themen/zweiterbildungsweg/exemplarische-angebote/pflichtschulabschluss.php> [Zugriff: 10.04.2020].

Buber-Ennser I., Kohlenberger J., Rengs B., Al Zalak Z., Goujon A., Striessnig E., Potanćoková M., Gisser R., Testa M.R. \& Lutz W. (2016), Human Capital, Values, and Attitudes of Persons Seeking Refuge in Austria in 2015. PLOS ONE 11 (9), pp. 1-29.

Buber-Ennser I., Kohlenberger J. \& Rengs B. (2019), Was wissen wir über die Geflüchteten, die 2015 zu uns kamen?. In: J. Kohlbacher \& M. Six-Hohenbalken (eds.), Die lange Dauer der Flucht - Analysen aus Wissenschaft und Praxis. ISR-Forschungsberichte, Band 49. Wien, Verlag der Österreichischen Akademie der Wissenschaften, pp. 109-129.

Bundesministerium für Arbeit, FAmilie und Jugend \& Bundesministerium für Bildung, Wissenschaft UND Forschung (o. J.), Schulbuchaktion online. $<$ https://www.schulbuch aktion.at/index.html> [Zugriff: 28.07.2020].

BUNDESMINISTERIUM FÜR BILDUNG (eds.) (2017) Flüchtlingskinder und-jugendliche an österreichischen Schulen. Beilage zum Rundschreiben Nr. 21/2017, Wien. <https://bildung.bmbwf. gv.at/ministerium/rs/2017_21.html> [Zugriff: 10.07.2019].

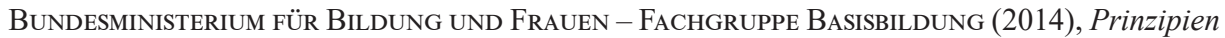
und Richtlinien für Basisbildungsangebote. <https://www.alphabetisierung.at/wp-content/ uploads/2017/08/Prinzipien_Richtlinien_Basisbildung_endg_141.pdf> [Zugriff: 01.04. 2020].

Bundesministerium für Bildung, Wissenschaft und Forschung (2018), Deutschförderklassen und Deutschförderkurse. Leitfaden für Schulleiterinnen und Schulleiter. Wien. $<$ https://bildung.bmbwf.gv.at/schulen/unterricht/ba/deutschfoerderklassen.pdf?6hwy6c > [Zugriff: 16.07.2019].

BUNDESMINISTERIUM FÜr BILDUNG, WisSENSCHAFT UNd ForsChUNG (ed.; Verfasserin: Gouma A.) (2019), Der muttersprachliche Unterricht in Österreich. Statistische Auswertung für das Schuljahr 2018/19. Informationsblätter zum Thema Migration und Schule, 5/2020. Wien. $<$ https://pubshop.bmbwf.gv.at/index.php?rex_media_type=pubshop_download\&rex_ media_file=200511_mustatistik_18_19.pdf $>$ [Zugriff: 11.08.2020].

BUNDESMINISTERIUM FÜR INNERES (2019), Asylstatistik 2019. <https://www.bmi.gv.at/301/Statis tiken/files/Jahresstatistiken/Asyl-Jahresstatistik_2019.pdf.> [Zugriff: 26.03.2020].

BundeSministerium Für UnTerRicht, Kunst Und Kultur (2013), Handreichung zum Pflichtschulabschluss: pädagogisch-didaktische Überlegungen. $<$ https:/erwachsenenbildung.at/ downloads/bildungsinfo/materialien_psa_fuer_rueckmeldung/Paedagogik_Didaktik.pdf.> [Zugriff: 10.04.2020].

Cantat C. \& Feischmidt M. (2019), Conclusion: Civil Involvement in Refugee Protection Reconfiguring Humanitarianism and Solidarity in Europe. In: FeISCHMIDT, M., PrIEs L. \& Cantat C. (eds.), Refugee Protection and Civil Society in Europe. Cham, Switzerland, Palgrave Macmillan, pp. 379-399.

Daschner P. (2017), Flüchtlinge in der Schule. Daten, Rahmenbedingungen und Perspektiven. Ein Überblick. In: McElvany N., Jungermann A., Bos W. \& Holtappels H.G. (eds.), Ankommen in der Schule: Chancen und Herausforderungen bei der Integration von Kindern und Jugendlichen mit Fluchterfahrung. IFS-Bildungsdialoge Band 1, Münster, Waxmann Verlag GmbH, pp. 11-25. 
De Cilla R. \& Dorostkar N. (2014), Integration und/durch Sprache. In: Dahlvik J., ReinPrecht C. \& Sievers W. (eds.), Migration und Integration: wissenschaftliche Perspektiven aus Österreich: Jahrbuch 2/2013. Göttingen, Vandenhoeck \& Ruprecht unipress, pp. 143-162.

De Jong S. \& AtaÇ I. (2017), Demand and Deliver: Refugee Support Organisations in Austria. Social Inclusion 5 (3), pp. 28-37.

El-MafaAlani A. \& Massumi M. (2019), Flucht und Bildung: frühkindliche, schulische, berufliche und non-formale Bildung. State-of-Research Paper 08a, Verbundprojekt Flucht: ,Forschung und Transfer', Osnabrück, Institut für Migrationsforschung und interkulturelle Studien (IMIS) der Universität Osnabrück / Bonn: Bonn International Center for Conversion (bicc). $<$ https://flucht-forschung-transfer.de/schulebildungausbildung/> [Zugriff: 28.05.2020].

ENTWICKLUNGSPARTNERSCHAFT „MEHRSPRACHIG VIELFÄLTIG“(2014), Mehrsprachigkeit im Basisbildungsunterricht - Materialien und Aktivitäten für jugendliche und junge erwachsene Migrant innen. <https://www.vhs.at/meviel handreichung/downloads/MEVIEL Handrei chung.pdf $>$ [Zugriff: 01.04.2020].

ERwachsenenbildung.AT. (o. J.), Nachholung des Pflichtschulabschlusses. <https://erwachse nenbildung.at/bildungsinfo/zweiter_bildungsweg/nachholung_pflichtschulabschluss.php.> [Zugriff: 30.03.2020].

ESSER H. (2001), Integration und ethnische Schichtung. < http://www.mzes.uni-mannheim.de/ publications/wp/wp-40.pdf > [Zugriff: 24.08.2019].

Eurostat (2019), Asylum applications (non-EU) in the EU-28 Member States, 2008-2018. $<$ https://ec.europa.eu/eurostat/statistics-explained/index.php/Asylum_statistics.> [Zugriff: 08.05.2019].

Evers A. \& Klie A.W. (2018), Flüchtlinge und Engagement. In: Klie T. \& KLIE A.W. (eds.), Engagement und Zivilgesellschaft. Expertisen und Debatten zum Zweiten Engagementbericht. Wiesbaden, Springer SV, pp. 513-546.

FASsin D. (2011), Policing Borders, Producing Boundaries. The Governmentality of Immigration in Dark Times. Annual Review of Anthropology 40 (1), pp. 213-226.

FASSMANN H. (о.J.), Integrationsindikatoren des Nationalen Aktionsplans für Integration. Begriffe - Beispiele - Implementierung. <https:/www.bmeia.gv.at/fileadmin/user_upload/Zentrale/ Integration/NAP/NAP _ indikatoren.pdf $>$ [Zugriff am 22.05.2019].

FASSMANN H. (2008), Integration und Integrationspolitische Leitlinien. In: LEIBETSEDER B. \& WEIDENHOLZER J. (eds.): Integration ist gestaltbar - Strategien erfolgreicher Integrationspolitik in Städten und Regionen. Wien, Braumüller, pp. 1-15.

FASSMANN H. (2010), Integrationsindikatoren des Nationalen Integrationsplanes für Integration. Bundesministerium für europäische und internationale Angelegenheiten. <https://www. bmeia.gv.at/fileadmin/ZZZ_Medien_alt/bmeia/media/Integration/NAP/NAP_Indikatoren. pdf> [Zugriff: 12.03.2020].

Fleischmann L. \& Steinhilper E. (2017), The Myth of Apolitical Volunteering for Refugees: German Welcome Culture and a New Disposif of Helping. Social Inclusion 5 (3), pp. 17-27.

FrIESE H. (2017), Flüchtlinge: Opfer - Bedrohung - Helden: Zur politischen Imagination des Fremden. Bielefeld, transcript Verlag.

FrIESE H. (2019), Framing Mobility. Refugees and the Social Imagination. In: KoHLBACHER J. \& Six-Hohenbalken M. (eds.), Die lange Dauer der Flucht-Analysen aus Wissenschaft und Praxis. ISR-Forschungsbericht 49. Wien, Verlag der österreichischen Akademie der Wissenschaften, pp. 25-42. 
GATT S. (2014), Sprachenpolitik politisch kommuniziert: Symbolische Instrumentalisierung zwischen Inklusion und Exklusion. In: DAhLviK J., ReinPrecht C. \& Sievers W. (eds.), Migration und Integration: wissenschaftliche Perspektiven aus Österreich: Jahrbuch 2/2013. Göttingen, Vandenhoeck \& Ruprecht unipress, pp. 163-180.

Gatterbauer M. (2019), Die lange Dauer der Flucht - Das Ankommen erleichtern durch die Begleitung des Arbeitsmarktservice bis hin zur Arbeitsmarktintegration. In: KOHLBACHER J. \& Six-Hohenbalken M. (eds.), Die lange Dauer der Flucht-Analysen aus Wissenschaft und Praxis. ISR-Forschungsberichte, Band 49. Wien, Verlag der Österreichischen Akademie der Wissenschaften, pp. 131-141.

GEMEINDE ST. ANDRÄ-WÖRDERn (o.J.), Zahlen \& Fakten. <https://www.staw.at/Unsere Gemeinde/Unser_Ort/Zahlen_Fakten.> [Zugriff: 07.08.2019].

GEMEINDE St. ANDRÄ-WÖRDERn (2016), Sitzungsprotokoll 02.12.2016. <https://www.staw.at// system/web/GetDocument.ashx?fileId=1318217> [Zugriff: 20.07.2020].

GeNFER FlÜCHTLINGSKONVENTION - Abkommen über die Rechtsstellung der Flüchtlinge. $<$ https:// www.unhcr.org/dach/wp-content/uploads/sites/27/2017/03/GFK_Pocket_2015_RZ_final_ ansicht.pdf.> [Zugriff: 26.04.2019].

Gesemann F. \& Roth R. (eds.) (2018), Handbuch lokale Integrationspolitik. Wiesbaden, Springer.

Gogolin I. (2008 [1994]), Der monolinguale Habitus der multilingualen Schule. Münster, Waxmann.

Gries J., Lindenau M., MaAz K. \& Waleschkowiski U. (2005), Bildungssysteme in Europa. Kurzdarstellungen. Institut für Sozialforschung, Informatik und Soziale Arbeit (ISIS Berlin e. V.), Berlin.

Griesser D. (2017), Migration als Strategie gegen die Landflucht. Der Standard, 22.01.2017. $<$ https://www.derstandard.at/story/2000050902734/migration-als-strategie-gegen-dielandflucht.> [Zugriff: 07.07.2019].

GruBER O. (2017), Integration statt Separation. Die Beschulung von Flüchtlingskindern und -jugendlichen. $A \& W$ blog, 13.09.2017. $<\mathrm{https}$ ://awblog.at/integration-statt-separation-diebeschulung-von-fluechtlingskindern-und-jugendlichen/> [Zugriff: 22.07.2020].

Gruber O. (2018), Diversität und Integration im Schulsystem. Empirische Befunde zur Ungleichheit und ihre bildungspolitischen Konsequenzen. GW-Unterricht 151 (3/2018), pp. 5-19.

Grzymala-Kazlowska A. \& Phillimore J. (2018), Introduction: Rethinking Integration. New Perspectives on Adaptation and Settlement in the Era of Super-Diversity. Journal of Ethnic and Migration Studies 44 (2), pp, 179-196.

Hackl-Schuberth K. (2014), Den Pflichtschulabschluss „erwachsenengerecht“ nachholen. Mehr Anschluss durch Abschluss? Magazin erwachsenenbildung.at. 21 (1), pp. 117-123. $<$ https://erwachsenenbildung.at/magazin/14-21/meb14-21.pdf $>$ [Zugriff: 03.09.2020]

HAgenthaler - SPÖ Zeitung FÜR ST. ANDRÄ-WÖRDERn (2016), Danke für die gute Betreunng! 03/2016, p. 9.

Han-Broich M. (2012), Ehrenamt und Integration. Die Bedeutung sozialen Engagements in der (Flüchtlings-)Sozialarbeit. Wiesbaden: Springer VS.

Heckmann F. (2003), From Ethnic Nation to Universalistic Immigrant Integration Germany. In: HeCKMANN F. \& SCHNAPPER D. (eds.), The Integration of Immigrants in European Societies National Differences and Trends of Convergence. Stuttgart, Lucius \& Lucius, pp. 45-78.

Heinemann A.M.B. (2017), The Making of ,Good Citizens': German Courses for Migrants and Refugees. Studies in the Education of Adults 49 (2), pp. 177-195. 
Heintze A. (2017), Chancen und Herausforderungen aus Sicht der Bildungsadministration. In: McElvany N., Jungermann A., Bos W. \& Holtappels H.G. (eds.), Ankommen in der Schule: Chancen und Herausforderungen bei der Integration von Kindern und Jugendlichen mit Fluchterfahrung. IFS Bildungsdialoge, 1, Münster, New York, Waxman, pp. 185-190.

Hemayat - Betreuungszentrum für Folter- und Kriegsüberlebende (2013), Krieg und Folter im Asylverfahren: eine psychotherapeutische und juristische Studie (Zusammenfassung). $<$ http://www.hemayat.org/fileadmin/user_upload/Zusammenfassung.pdf $>$ [Zugriff: 11.05.2020].

Hess S. \& Moser J. (2009), Jenseits der Integration. Kulturwissenschaftliche Betrachtungen einer Debatte. In: Hess S, Binder J. \& Moser J. (eds.), No Integration?! Kulturwissenschaftliche Beiträge zur Integrationsdebatte in Europa. Bielefeld, transcript Verlag, pp. 11-25.

Hess S., Kasparek B., Kron S., Rodatz M., Schwertl M. \& Sontowski S. (2017), Der lange Sommer der Migration. Krise, Rekonstitution und ungewisse Zukunft des europäischen Grenzregimes. In: Hess S., Kasparek B., Kron S., Rodatz M., Schwertl M. \& Sontowski S. (eds.), Der lange Sommer der Migration. Grenzregime III. Hamburg, Assoziation A e.V., pp. 6-24.

Holmes S.M. \& CASTAÑEda H. (2016), Representing the „European refugee crisis” in Germany and beyond: Deservingness and difference, life and death. American Ethnologist, 43 (1), pp. 12-24.

Internationale Organisation für Migration (IOM) (2010), World Migration Report 2010: The Future of Migration: Building Capacities for Change. <http://publications.iom.int/ system/files/pdf/wmr_2010_english.pdf?language=en.> [Zugriff: 24.08.2019].

Islamic Republic of Afghanistan - Central Statistics Organization (2018), Afghanistan Living Conditions Survey 2016-17. Kabul. <https://reliefweb.int/sites/reliefweb.int/files/ resources/alcs_2017.pdf> [Zugriff: 02.09.2020].

IzQuierdo R., Makhashvili N., DrožĐek B. \& Wenzel T. (2018), Mental Health and Well-Being of the Staff Supporting Refugees: How to Deal with Risks? In: Wenzel T. \& DrožĐeK B. (eds.), An Uncertain Safety - Integrative Health Care for the $21^{\text {st }}$ Century Refugees. Cham, Springer, pp. 363-386.

IzQuierdo R., Makhashvili N., DrožĐek B. \& Wenzel T. (2018), Mental Health and Well-Being of the Staff Supporting Refugees: How to Deal with Risks? In: Wenzel T. \& DrožĐeK B. (eds.), An Uncertain Safety - Integrative Health Care for the $21^{\text {st }}$ Century Refugees. Cham, Springer, pp. 363-386.

INBAS-SOZIALFORSCHUNG (2018), Kooperation von Haupt- und Ehrenamtlichen in der Arbeit mit Geflüchteten. Bestandsaufnahme und Handlungsempfehlungen. Frankfurt/Main. < https:// www.inbas-sozialforschung.de/projekte/kooperation-von-haupt-und-ehrenamtlichen-inder-arbeit-mit-gefluechteten.html> [Zugriff: 07.08.2020].

Jenewein F. (2014), Basisbildung und Nachholen des Pflichtschulabschlusses. Konkrete Bildungsmaßnahmen in Tirol im Rahmen der „Initiative Erwachsenenbildung“. Magazin erwachsenenbildung.at 21 (1), pp. 44-49. <https://erwachsenenbildung.at/magazin/14-21/ meb14-21.pdf> [Zugriff: 03.09.2020].

KALAYCI E. (2009), ,,Integrierts euch!? “: Grundlagen, Hürden und Vision im Integrationsprozess von MigrantInnen. Wien, Planet Verlag.

KAPELLER L. (2018), „Mehr Integration und weniger Unterstützung, das geht nicht“. Kurier, 21.03.2018. $<$ https://kurier.at/politik/inland/mehr-integration-und-weniger-unterstuetzungdas-geht-nicht/400008387> [Zugriff: 30.08.2020]. 
Karakayali S. (2017), 'Infra-Politik' der Willkommensgesellschaft. Forschungsjournal Soziale Bewegungen 30 (3), pp. 16-24.

Karakayali S. (2018), Ehrenamtliches Engagement für Geflüchtete in Deutschland. State-ofResearch Papier 09. Verbundprojekt ,Flucht: Forschung und Transfer', Osnabrück: Institut für Migrationsforschung und interkulturelle Studien (IMIS) der Universität Osnabrück / Bonn: Internationales Konversionszentrum Bonn (BICC). <https://flucht-forschungtransfer.de/wp-content/uploads/2017/05/SoR-09-Karakayali.pdf> [Zugriff: 02.09.2020].

Karakayali S. \& KLeist, O. (2015), EFA-Studie: Strukturen und Motive der ehrenamtlichen Flüchtlingsarbeit in Deutschland, 1. Forschungsbericht: Ergebnisse einer explorativen Umfrage von November/Dezember 2014. Berliner Institut für empirische Integrations- und Migrationsforschung (BIM), Humboldt-Universität zu Berlin. $<$ https://www.bim.hu-berlin. de/de/publikationen/2015/strukturen-und-motive-der-ehrenamtlichen-fluechtlingsarbeitefa-in-deutschland/> [Zugriff: 13.08.2019].

Kastner M. (2016), Alphabetisierung und Basisbildung für Erwachsene. $<$ https://erwachsenen bildung.at/images/themen/dossier/ebooks/dossier-basisbildung.pdf $>$ [Zugriff: 01.04.2020].

KLEIST J. O. (2017), Bildungsarbeit von Ehrenamtlichen in der Flüchtlingsarbeit in Deutschland. ZEP: Zeitschrift für internationale Bildungsforschung und Entwicklungspädagogik 40 (1), pp. 27-31.

KLINGE D. (2017), Häufig gestellte Fragen zu Flucht, Asyl und Bildung in Österreich. Magazin erwachsenenbildung.at 31 (1), pp. 70-75. $<$ https://erwachsenenbildung.at/magazin/17-31/ meb17-31.pdf> [Zugriff: 07.04.2020].

KLOUBERT T. (2020), Einleitung der Herausgeberin. In: Kloubert T. (ed.): Erwachsenenbildung und Migration. Internationale Kontexte und historische Bezüge. Wiesbaden, Springer VS, pp. $1-10$.

KöGLER B. (2008), Und die Staatsbürgerschaft als Belohnung ...: Lebensbedingungen und Integration von anerkannten Flüchtlingen im Industrieviertel. In: ScHMidinger T. (ed.), Vom selben Schlag - Migration und Integration im niederösterreichischen Industrieviertel. Wiener Neustadt, Verein Alltag Verlag, pp. 173-180.

Kohlbacher J. \& Six-Hohenbalken M. (eds.) (2019), Die lange Dauer der Flucht - Analysen aus Wissenschaft und Praxis. Wien, Verlag der österreichischen Akademie der Wissenschaften.

Kohlbacher J., Rasuly-Paleczek G., Hackl A. \& Bauer S. (2017), Endbericht: Wertehaltungen und Erwartung von Flüchtlingen in Österreich. <https://www.bundeskanzleramt. gv.at/service/publikationen-aus-dem-bundeskanzleramt/publikationen-zu-integration/ studien-zum-thema.html> [Zugriff: 03.06.2020].

Korntheuer A. (2016), Die Bildungsteilhabe junger Flüchtlinge. Faktoren von Inklusion und Exklusion in München und Toronto. Bildung in Umbruchsgesellschaften, 13. Münster \& New York, Waxmann.

Lachmayr N., LeitgöB H. \& Bacher J. (2011), Expertise zur sozialen Selektion beim Bildungszugang: Schwerpunkt MIGRATION. Österreichisches Institut für Berufsbildungsforschung (öibf), Wien.

LANGTHALER H. (2016), Bildung für Flüchtlingskinder. asyl aktuell, 1/2016, pp. 2-8. <https:// www.asyl.at/adincludes/dld.php?datei=154.04.ma,bildung_fr_flchtlingskinder.pdf> [Zugriff: 28.10.2019].

Langthaler H. \& Trauner H. (2009), Das österreichische Asylregime unter besonderer Berücksichtigung der Rolle zivilgesellschaftlicher Organisationen. SWS-Rundschau 49 (4), pp. 446-467. 
LASSNIGG L. (2015), Das ,österreichische Modell` der Bildungsungleichheit: Hohe soziale Reproduktion, starke Umverteilung, politische Polarisierung. Reihe Soziologie 109, Institut für Höhere Studien, Wien.

LiChTENWAgNer H. (2019), Bedingungen und Herausforderungen der Arbeitsmarktintegration von Geflüchteten im ländlichen Raum: eine qualitative Studie im Südburgenland. Masterarbeit, Universität Wien.

Lichtenwagner H., Nechansyk S., Punz, S \& Schwarzbauer A. (2019), Entwicklungen und Strukturen des ehrenamtlichen Engagements für Geflüchtete seit 2015. Vier Beispiele aus österreichischen Regionen. In: KohlBacher J. \& Six-Hohenbalken M. (eds.), Die lange Dauer der Flucht - Analysen aus Wissenschaft und Praxis. ISR-Forschungsbericht 49. Wien, Verlag der Österreichischen Akademie der Wissenschaften, pp. 265-293.

LidzBa S. (2018), Freiwilligenkoordination in der Geflüchtetenhilfe. Kommunale Kooperationsmodelle. In: W. Schiffauer, A. Eilert \& M. Rudloff (eds.), So schaffen wird das - eine Zivilgesellschaft im Aufbruch. Bedingungen für die nachhaltige Projektarbeit mit Geflüchteten. Eine Bilanz. Bielefeld, Transcript Verlag, pp. 273-287.

LinTNER C. (2017), Ressourcen auf dem Abstellgleis? Die Anerkennung von Kompetenzen, Lebens- und Arbeitserfahrung im Migrationskontext. Magazin erwachsenenbildung.at. 31 (1), pp. 49-57. <https://erwachsenenbildung.at/magazin/17-31/meb17-31.pdf> [Zugriff: 07.04.2020].

LUCIAK M. \& BiNDER S. (2010), Informationen und Anregungen zur Umsetzung des Unterrichtsprinzips „Interkulturelles Lernen“. Ein Handbuch für den Bereich allgemeinbildende Pflichtschulen und allgemeinbildende höhere Schulen. Austrian Studies in Social Anthropology (ASSA), Online-Journal des Vereins der AbsolventInnen des Instituts für Kultur- und Sozialanthropologie der Universität Wien. < https://www.univie.ac.at/ alumni.ksa/wp-content/uploads/text-documents/ASSA/ASSA-SN-2010-01.pdf> [Zugriff: 02.09.2020].

LütJe-Klose B., Miller S., Schwab S. \& Streese B. (eds.) (2017), Inklusion: Profil für die Schul- und Unterrichtsentwicklung in Deutschland, Österreich und der Schweiz. Theoretische Grundlagen - Empirische Befunde - Praxisbeispiele. Münster, Waxmann Verlag.

Machold I. \& Dax T. (2016), Migration und Integration: Anstoß zur sozio-kulturellen Veränderung ländlicher Regionen durch internationale Migration. Europa Regional, 24 (3-4), pp. $62-76$.

Markom C. (2009), Geschichte der Migrationsforschung. In: Six-Hohenbalken M. \& Tošić J. (eds.), Anthropologie der Migration - Theoretische Grundlagen und interdisziplinäre Ansätze. Wien, Facultas, pp. 29-49.

Martín I., Arcarons A., Aumüller J., Bevelander P., Emilsson H., Kalantaryan S., Maclver A., Mara I., Scalettaris G., Venturini A., Vidovic H., van der Welle H., Windisch M., WolfFberg R. \& Zorlu A. (2016), From Refugees to Workers. Mapping Labour Market Integration Support Measures for Asylum Seekers and Refugees in EU Member States. Volume 1. Bertelsmann Stiftung, Gütersloh. <https://www.bertelsmann-stiftung. de/fileadmin/files/BSt/Bibliothek/Doi_Publikationen/Studie_NW_From_Refugees_to_ Workers_Vol1.pdf $>$ [Zugriff: 02.10.2019]].

McElvany N., Jungermann A., Bos, W. \& Holtappels H.G. (eds.) (2017), Ankommen in der Schule: Chancen und Herausforderungen bei der Integration von Kindern und Jugendlichen mit Fluchterfahrung. IFS Bildungsdialoge, 1, Münster, New York, Waxman.

MenL P. (ed.) (2017), Aufnahme und Integration von Geflüchteten in ländliche Räume: Spezifika und (Forschungs-)herausforderungen. Beiträge und Ergebnisse eines Workshops am 6. 
und 7. März 2017 in Braunschweig. Thünen Report, 53. Braunschweig, Johann Heinrich von Thünen-Institut. <https://literatur.thuenen.de/digbib_extern/dn059278.pdf> [Zugriff: 07.08.2020].

MoBAyyen M. (2013), Bildungsbenachteiligung und soziale Exklusion junger AsylwerberInnen ab dem Pflichtschulalter in Österreich. Diplomarbeit, Universität Wien.

Mourão-Permoser J. \& Rosenberger S. (2012), Integration Policy in Austria. In: Frideres J. \& Biles J. (eds.), International Perspectives - Integration and Inclusion. (Queen's Policy Studies Series). Kingston, McGill-Queen‘s University Press, pp. 39-58.

Nechansky S. (2019), Integration von Asylwerber/innen und Asylberechtigten in den Arbeitsmarkt: Politik und Maßnahmen in Oberösterreich. Masterarbeit, Universität Wien.

Oberwimmer K., Vogtenhuber S., Lassnigg L. \& Schreiner C. (eds.) (2019), Nationaler Bildungsbericht Österreich 2018, Band 1: Das Schulsystem im Spiegel von Daten und Indikatoren. Bundesministerium für Bildung, Wissenschaft und Forschung / Bundesinstitut für Bildungsforschung, Innovation \& Entwicklung des österreichischen Schulwesens.

OEAD (2014), Das österreichische Bildungssystem. < https://www.bildungssystem.at/> [Zugriff: 09.06.2020].

OECD (2016), Migrationshintergrund, Schülerleistungen und Einstellungen gegenüber Naturwissenschaften. In: OECD (ed.), PISA 2015 Results (Volume I): Excellence and Equity in Education. Paris, OECD Publishing, pp. 261-284.

Ohlinger R., Schweiger R. \& Veyhl L. (2017), Auf dem Weg zur Flüchtlingsintegration in ländlichen Räumen: Ergebnisse einer Bedarfsanalyse in sieben Landkreisen. RobertBosch- Stiftung. <https://www.bosch-stiftung.de/de/publikation/auf-dem-weg-zur-fluecht lingsintegration-laendlichen-raeumen-ergebnisse-einer> [Zugriff: 07.08.2020].

Oesterreich.gv.AT (2020), Allgemeines zum Asyl. <https://www.oesterreich.gv.at/themen/leben in_oesterreich/asyl/Seite.3210001.html.> [Zugriff: 26.03.2020].

ÖSTERREICHISChe Jungarbeiterbewegung (o.J.), Über die ÖJAB. <https://www.oejab.at/ueberdie-oejab/geschichte.> [Zugriff: 09.06.2019].

ÖSTERREICHISCHE RAUMORDNUNGSKONFERENZ (2019), Kleinräumige Bevölkerungsprognose für Österreich 2018 bis 2040 mit einer Projektion bis 2060 und Modellfortschreibung bis 2075 (ÖROK-Prognose). <http://www.forschungsnetzwerk.at/downloadpub/Bericht_BevProgno se_2018.pdf> [Zugriff: 04.06.2020].

PANZENBöCK M. (2017), Mittels sozialarbeiterischer Beratung die Möglichkeit für konzentriertes Lernen schaffen. PROSA - Projekt Schule für Alle! Magazin erwachsenenbildung.at 31 (1), pp. 76-83. <https://erwachsenenbildung.at/magazin/17-31/meb17-31.pdf> [Zugriff: 07.04.2020].

Perching B. (2010), Migration, Integration und Staatsbürgerschaft - was taugen die Begriffe noch? In: LANGTHALER H. (ed.), Integration in Österreich: sozialwissenschaftliche Befunde. Wien, Studien-Verlag, pp. 13-32.

Phillimore, J. (2012), Implementing Integration in the UK: Lessons for Integration Theory, Policy and Practise. Policy \& Politics, 40 (4), pp. 525-545.

PILz S. (2016), Schulentwicklung als Antwort auf Heterogenität und Ungleichheit. Organisation und Pädagogik, 25, Wiesbaden, Springer VS.

Plutzar V. (2010), Sprache als „Schlüssel“ zur Integration? Eine kritische Annäherung an die österreichische Sprachenpolitik im Kontext von Migration. In: LANGTHALER H. (ed.), Integration in Österreich: sozialwissenschaftliche Befunde. Wien, Studien-Verlag, pp. 123-142. 
Preuss B. (2018), Inklusive Bildung im schulischen Mehrebenensystem. Behinderung, Flüchtlinge, Migration und Begabung. Wiesbaden, Springer VS.

PrIES L. (2014), Integration als Eröffnung gesamtgesellschaftlicher Teilhabechancen. In: PRIES L. (ed.): Dokumentation der Tagung ,,Migrations- und Integrationspolitik heute. “ Rat für Migration, Berlin, 22. Nov. 2013, pp. 46-60.

Pries L. (2019), Introduction: Civil Society and Volunteering in the So-Called Refugee Crisis of 2015 - Ambiguities and Structural Tensions. In: Feischmidt M., Pries L. \& Cantat C. (eds.), Refugee Protection and Civil Society in Europe. Cham, Palgrave Macmillan, pp. $1-23$.

Ravn S., Mahieu R., Belloni M. \& Timmerman C. (2020), Shaping the „Deserving Refugee”: Insights from a Local Reception Programme in Belgium. In: GLORIUS B. \& DoOMERNIK J. (eds.), Geographies of Asylum in Europe and the Role of European Localities. IMISCOE Research Series. Cham, Switzerland, Springer Open, pp. 135-153.

Ryan L. (2018), Differentiated Embedding: Polish Migrants in London Negotiating Belonging Over Time. Journal of Ethnic and Migration Studies 44 (2), pp. 233-251.

ROTHWEILER M. \& RUBERG T. (2011), Der Erwerb des Deutschen bei Kindern mit nichtdeutscher Erstsprache. Sprachliche und außersprachliche Einflussfaktoren. WiFF Expertisen, 12, München, Deutsches Jugendinstitut e. V.

ReITTER V. (2017), Akzeptanz mit Vorbehalt. Eine Untersuchung internationaler Immigration im ländlichen Raum Österreichs. Masterarbeit, Universität Wien.

Rosenberger S. (2011), Integration von AsylwerberInnen? Zur Paradoxie individueller Integrationsleistungen und staatlicher Desintegration. In: DahlviK J., FASSMAnN H. \& Sievers W. (eds.), Migration und Integration - wissenschaftiche Perspektiven aus Österreich. Jahrbuch 1. Migrations- und Integrationsforschung. Göttingen, Vandenhoeck \& Ruprecht unipress, pp. 91-106.

RÜTZeL J. (2010), Kompetenz und Diagnostik: Fördern, Auslesen, Bilden. Vortrag beim Berufspädagogischen Kolloquium am 03. November 2010 an der TU Darmstadt.

Scheibelhofer P. (2017), 'It Won't Work Without Ugly Pictures': Images of Othered Masculinities and the Legitimisation of Restrictive Refugee-Politics in Austria. International Journal for Masculinity Studies, 12 (2), pp. 96-111.

SCherR A. \& InAN Ç. (2018), Leitbilder in der politischen Debatte: Integration, Multikulturalismus und Diversität. In: GeSEMANN F. \& Roth R. (eds.), Handbuch lokale Integrationspolitik. Wiesbaden: Springer, pp. 201-226.

Schmidinger T. (2010), Migration und Integration. In: Lamgthaler H. (ed.), Integration in Österreich: sozialwissenschaftliche Befunde. Wien, Studien-Verlag, pp. 33-41.

Schmidinger T. \& Gysi S. (2008), „Eine lange Weile Langeweile“: Struktur und Lebensbedingungen von AsylwerberInnen im Industrieviertel. In: SCHMIDINGER T. (ed.): Vom selben Schlag - Migration und Integration im niederösterreichischen Industrieviertel. Wiener Neustadt, Verein Alltag Verlag, pp. 133-148.

SchUG (1986), Bundesgesetz über die Ordnung von Unterricht und Erziehung in den im Schulorganisationsgesetz geregelten Schulen (Schulunterrichtsgesetz - SchUG), StF: BGBl. Nr. $472 / 1986$

Schule MehrSPRAChig (2020), Muttersprachlicher Unterricht. <https://www.schule-mehr sprachig.at/muttersprache/service/schulen> [Zugriff: 11.08.2020].

SCHÜHRER A-K. (2019), Migration und Engagement. Zwischen Anerkennung, Lebensbewältigung und sozialer Inklusion. Edition Centaurus - Perspektiven Sozialer Arbeit in Theorie und Praxis. Wiesbaden, Springer VS. 
Sen A. (1999), Ökonomie für den Menschen. Wege zu Gerechtigkeit und Solidarität in der Marktwirtschaft. München, Carl Hanser.

Simsa R. (2017), Leaving Emergency Management in the Refugee Crisis to Civil Society? The Case of Austria. Journal of Applied Security Research 12 (1), pp. 78-95.

Slama M. (2012), Von der Kolonialzeit bis ins post-9/11 Indonesien. Zur Integration arabischer Migranten in eine muslimische Mehrheitsgesellschaft. In: Fassmann H. \& DahlviK J. (eds.), Migration und Integration - wissenschaftliche Perspektiven aus Österreich: Jahrbuch 1/2011. Göttingen, Vandenhoeck \& Ruprecht unipress, pp. 265-279.

SOS Mitmensch (2017), Deutschkurse für Asylsuchende - Ein Bundesländervergleich: Recherche von SOS Mitmensch für den Zeitraum Oktober 2016-Jänner 2017. <https://www.sosmit mensch.at/d1/skprJKJKLmkJqx4KJK/Deutschkurse_fuer_Asylsuchende_Bundeslaender vergleich_SOS_Mitmensch_Jaenner2017_neu_mitVorarlberg.pdf.> [Zugriff: 25.03.2020].

SOS Mitmensch (2019), Integrationspolitik auf dem Rückzug? - Expertinnen und Experten analysieren integrative und desintegrative Maßnahmen der Bundesregierung. <https:// www.sosmitmensch.at/d1/LlnLJKJKnooJqx4KJK/SOS_Mitmensch_ExpertInnen_ Bericht_Integrationspolitik_Maerz2019.pdf.> [Zugriff: 25.03.2020].

StATISTIK AUSTRIA (2016), Urban-Rural-Typologie. <https://www.statistik.at/wcm/idc/ idcplg?IdcService=GET_PDF_FILE\&dDocName=108332.> [Zugriff: 29.06.2019].

Statistik Austria (2018), Zuordnungen - Urban-Rural-Typologie Statistik Austria - Gemeinden inkl. Bevölkerungszahl. <https://www.statistik.at/wcm/idc/idcplg?IdcService=GET NATIVE_FILE\&RevisionSelectionMethod=LatestReleased\&dDocName=108339.> [Zugriff: 29.06.2019].

Statistik Austria (2019), Asylanträge 2000-2018 nach Staatsangehörigkeit. <https://www. statistik.at/web_de/statistiken/menschen_und_gesellschaft/bevoelkerung/wanderungen/ asyl/022914.html $>$ [Zugriff: 01.09.2020].

Stegbauer C. (2011), Reziprozität: Einführung in soziale Formen der Gegenseitigkeit. Wiesbaden, VS Verlag für Sozialwissenschaften.

Steiner M., Egger-Steiner M. \& Baumegger D. (2018), Evaluation der Bildungsmaßnahmen für junge Flüchtlinge im Bereich Erwachsenenbildung. Institut für höhere Studien, Wien.

Stewart E. (2005), Exploring the Vulnerability of Asylum Seekers in the UK. Population, Space and Place 11 (6), pp. 499-512.

Strasser E. (2009), Was ist Migration? Zentrale Begriffe und Typologien. In: Six-HohenBalken M. \& Tošı́ J. (eds.): Anthropologie der Migration - Theoretische Grundlagen und interdisziplinäre Ansätze. Wien, Facultas, pp. 15-28.

TÄUBIG V. (2009), Totale Institution Asyl. Empirische Befunde zu alltäglichen Lebensführungen in der organisierten Desintegration. Weinheim/München, Juventa Verlag.

Tošić J., Kroner G. \& Binder S. (2009), Anthropologische Flüchtlingsforschung. In: SiX-HoHeNBALKen M. \& Toší́ J. (eds.), Anthropologie der Migration - Theoretische Grundlagen und interdisziplinäre Ansätze. Wien, Facultas, pp. 110-126.

UNHCR (2013), Facilitators and Barriers. Refugee Integration in Austria. Refugee Integration Capacity and Evaluation in Europe (RICE) <https://www.refworld.org/docid/5278dc644. html> [Zugriff: 11.04.2019].

UNHCR (2019), Global Trends - Forced Displacement in 2018. <https://www.unher.org/ 5d08d7ee7.pdf.> [Zugriff: 24.08.2019].

UNHCR ÖsterReICH (2016), Flucht und Trauma im Kontext Schule. Ein Handbuch für PädagogInnen. Wien. 
UNHCR Wien (2013), Reception Facility Monitoring 2013 - Flüchtlingshaus Greifenstein $(\ddot{O} J A B)$. Unveröffentlicht.

Valtonen K. (2004), From the Margin to the Mainstream: Conceptualizing Refugee Settlement Processes. Journal of Refugee Studies 14 (1), pp. 70-96.

VAn Dyк S. \& MisBach E. (2016), Zur politischen Ökonomie des Helfens. Flüchtlingspolitik und Engagement im flexiblen Kapitalismus. PROKLA 46 (183), pp. 205-227.

VAndeVoordt, R. \& Verschraegen, G. (2019), Subversive Humanitarianism and Its Challenges: Notes on the Political Ambiguities of Civil Refugee Support. In: Feischmidt M., Pries L. \& Cantat C. (eds.), Refugee Protection and Civil Society in Europe. Cham, Switzerland, Palgrave Macmillan, pp. 101-128.

Verein Deutsch.Fit (o.J.), Deutschkurs deutsch.fit - Das kostenlose Deutsch-Lehrmaterial für ehrenamtliche Lehrer. <https://deutsch.fit/Deutschkurs/> [Zugriff: 04.05.2020].

Vertovec, S. (2007), Super-Diversity and its Implications. Ethnic and Racial Studies, 30 (6), pp. 1024-1054.

Von Dewitz N. \& Massumi M. (2017), Schule im Kontext aktueller Migration. Rechtliche Rahmenbedingungen, schulorganisatorischer Modelle und Anforderungen an Lehrkräfte. In: McElvany N., Jungermann A., Bos W. \& Holtappels H.G. (eds.), Ankommen in der Schule: Chancen und Herausforderungen bei der Integration von Kindern und Jugendlichen mit Fluchterfahrung. IFS Bildungsdialoge, 1, Münster, New York, Waxman, pp. 27-40.

WAGNer G. (2019), Helfen und Reziprozität. Freiwilliges Engagement für Geflüchtete im ländlichen Raum. Zeitschrift für Soziologie 48 (3), pp. 226-241.

Weidinger T., Kordel S. \& Pohle P. (2017), Bleiben oder Gehen? Einflussfaktoren auf die Wohnstandortmobilität anerkannter Flüchtlinge in ländlichen Räumen am Beispiel des Bayrischen Waldes. Europa Regional, 24 (3-4), pp. 46-61.

WiLKINSON L. (2002), Factors Influencing the Academic Success of Refugee Youth in Canada. Journal of Youth Studies, 5 (2), pp. 173-193.

Wohlfarth, C. \& KolB, H. (2016), Fünf Jahre Integrationspolitik in Österreich. In: SVR (ed.), Forschungsbereich beim Sachverständigenrat deutscher Stiftungen für Integration und Migration (SVR) GmbH. Berlin, pp. 1-31.

\subsection{Empirisches Material}

Amtsleiter Gemeinde C (2018), Interview von Sandra Punz am 10.07.2018.

Asylberechtigter Syrien St. ANDrä-Wördern (2018), Interview von Andreas Schwarzbauer und Hazem Amin am 12.10.2018.

ASYlwerber Iran St. ANDRÄ-WÖrdern (2018), Interview von Andreas Schwarzbauer am 22.10.2018.

Bürgermeister Gemeinde A (2018), Interview von Sandra Punz am 30.05.2018.

Bürgermeister St. ANDRÄ-WÖRdern (2018), Interview von Andreas Schwarzbauer am 13.06.2018.

DaF/DaZ-Förderlehrerin VS \& NMS Gemeinde B (2018), Interview von Sandra Punz am 24.10.2018 
DaZ-Lernbegleiter Basisbildung NÖ, „Bildung hat Wert“ (2018), Interview von Sandra Punz am 14.11.2018.

DaZ-Trainerin Mentor (2018), Interview von Sandra Punz am 14.11.2018.

Diakon Pfarre St. Andrä vor dem Hagental (2018), Interview von Andreas Schwarzbauer am 05.09.2018.

Direktor NMS Gemeinde A (2018), Interview von Sandra Punz am 08.06.2018.

DireKTor NMS Gemeinde B (2018), Interview von Sandra Punz am 04.07.2018.

DireKTORIN NMS St. ANDRÄ-WÖRDERN (2018), Interview von Andreas Schwarzbauer am 07.06.2018.

Direktorin VS Gemeinde A (2018), Interview von Sandra Punz am 18.06.2018.

DirekTORIN VS St. ANDRÄ-WÖRdern (2018), Interview von Andreas Schwarzbauer am 24.05.2018.

Ehrenamtliche Deutschlehrerin St. Andrä-Wördern (2018), Interview von Andreas Schwarzbauer am 23.05.2018.

Ehrenamtliche UnterstütZerin Gemeinde A (2018), Interview von Sandra Punz am 15.11.2018.

Ehrenamtliche UnterstütZerin Gemeinde C (2018), Interview von Sandra Punz am 19.11.2018.

EHRENAMTLICHER UNTERSTÜTZER ST. ANDRÄ-WÖRDERN (2018), Interview von Andreas Schwarzbauer am 11.07.2018.

Gemeinderat für Sozial- Gesundheitswesen: Integration, Migration, Freizeit, VeranSTALTUNGen St. ANDRÄ-WÖRDERn (2018), Interview von Andreas Schwarzbauer am 24.04.2018.

Koordinatorin ehrenamtlicher Deutschkurs St. ANDRä-Wördern (2018), Interview von Andreas Schwarzbauer am 22.05.2018.

Leiter BACH Bildungszentrum (2018), Interview von Andreas Schwarzbauer am 02.07.2018.

Leiter BAuHof St. ANDRÄ-WÖrdern (2018), Interview von Andreas Schwarzbauer am 18.06.2018.

Leiter „GrenZenlos“-Solidarfonds (2018), Interview von Andreas Schwarzbauer am 06.06.2018.

LEITERIN „ST. ANDRÄ-WÖRDERN HILFT“ (2018), Interview von Andreas Schwarzbauer am 08.05.2018.

Mitarbeiterin Migrationsabteilung und Trainerin Basisbildung Rotes Kreuz Tulln (2018), Interview von Andreas Schwarzbauer, 06.06.2018.

Mitarbeiterin mobile Beratung Caritas Korneuburg (2018), Interview von Andreas Schwarzbauer am 26.07.2018.

Mitarbeiterin Projekt Missing Link/ZusammenReden der Caritas Wien (2018), Interview von Andreas Schwarzbauer am 29.06.2018.

Obmann „Willkommens-Verein“ Gemeinde A (2019), Interviewt von Sandra Punz am 11.01.2019.

Projektkoordinator und Lehrerin des Pflichtschulabschlusskurses WIFI (Gruppeninterview) (2018), Interview von Sandra Punz am 25.10.2018.

Punz S. (2018), Feldnotizen, 24.10.2018 
SChwARZBAuER A. (2018), Beobachtungsprotokoll muttersprachliche Interviews mit Geflüchteten in St. Andrä-Wördern, 22.10.2018.

Senior/InN/En-Lehrer/InNEN Gemeinde B (Gruppeninterview) (2018), Interview von Sandra Punz am 24.10.2018.

Sozialarbeiterin Diakonie - mobiler FlǘhtlingSDienst (2018), Interview von Sandra Punz am 26.06.2018.

Trainerin Pflichtschulabschlusskurs PROSA (2018), Interview von Andreas Schwarzbauer 24.11.2018. 